\title{
Zur Rolle der Geologie im interdiskursiven "crossing" der Kulturen der deutschsprachigen Literatur zwischen 1960 und 1980: Botho Strauß und Hans Lebert
}

\section{ALEŠ URVÁLEK}

DOI: https://doi.org/10.31577/WLS.2021.13.4.2

Eines der wenigen Pauschalurteile über die deutsche Literatur nach 1945, das man nicht so einfach vom Tisch wischen kann, betrifft den intellektuellen Bildungsprozess der Generationen, die sich in den 1950ern zu Wort gemeldet haben, um sich spätestens in den 1960er Jahren zu etablieren. Man wuchs überwiegend mit Theodor W. Adorno, Ernst Bloch, oder Walter Benjamin auf, somit intellektuell geprägt durch die Paradigmen des linken geschichtsphilosophischen Denkens. Warum man es tat, dürfte mit zumindest zwei kulturell bedingten Abstoßmechanismen zu erklären sein: erstens aus dem Unwillen vieler jüngerer Autoren und Autorinnen, sich mit den auf kollektives Beschweigen der Zeit vor 1945 sowie auf die relativ bald nach 1945 umgesetzte Integration aller Ex-Nazis hin ausgerichteten Praktiken abzufinden. Diesem zeit- und generationstypischen Protest gegen die als restaurativ bezeichneten 1950er Jahre, dessen Intensität allerdings manche Beteiligten nachträglich relativieren, wenn nicht ganz bestreiten (Enzensberger 1988, 251), ging noch eine frühere Geste der Negation voraus, die folgendes Zitat von Botho Strauß deutlich macht: Man habe, erinnert sich dieser 1944 geborene Autor an die Urszene seiner Lehrjahre, die "Augen aufgeschlagen als zum Bewußtsein kommender Mensch - und habe ein Blutbad vor [sich] gesehen. Es ließ sich im Grunde nicht begreifen. Also rettete man sich nach links" (Radix 1987, 214).

Der rettende Sprung nach links stellte, wollte man sich nicht an dem von den Rechten angerichteten Blutbad beteiligen, für diese Generationen somit die einzig denkbare und vertretbare Option dar. Soweit war er recht und gut. Doch nach und nach zeigte sich manchem und mancher, dass dieser Rettungsring eher einem Käfig gleichen würde, in den man nicht freiwillig geflüchtet ist, aus dem man sich daher recht schwer wird befreien können. Wo man im Zwangsreflex Zuflucht gefunden habe, sei man unfrei, dachten nun nicht wenige, und ob man sich bei diesem reflexartigen Sprung von rechts nach links retten, oder von links nach rechts absondern wollte, mache keinen Unterschied. Kurzum, spätestens Mitte der 1970er Jahre waren im linken intellektuellen Feld deutliche Ermüdungserscheinungen sichtbar, die - so die These - der Erkenntnis geschuldet sein dürften, dass der gesamte Rahmen, in dem statt Reflexion eher nur Reflexe walten, wenig taugt. Diese Erkenntnis führte wohl bald zum Unbehagen am gesamten geschichtsphilosophischen Paradigma, das - egal ob in seiner linken oder rechten Version - als zu eng empfunden wurde. 
Im Hinblick auf diese Prozesse könnten nicht nur Fragen nach deren intellektuellen Kontexten aufschlussreich sein, sondern auch die möglichen Alternativen jenseits des geschichtsphilosophischen Denkens. Zu untersuchen wäre dabei somit nicht nur der Wissensstand der Literaten und Literatinnen, sondern vor allem die Formen der interdiskursiven Bildungs- und Transformationsprozesse, die der damalige Wissensstand in literarischen und essayistischen Texten durchmachte. Exemplarisch soll dies am Rumor, einem bisher wenig interpretierten Roman von Botho Strauß untersucht werden, dem folglich als Kontrastfolie mit Hans Leberts Die Wolfshaut ein bereits 1960 verfasster Roman eines österreichischen Autors zur Seite gestellt wird. Von den drei bereits profilierten Ansätzen einer kulturwissenschaftlich orientierten Germanistik, der es hierbei in interdisziplinärer Perspektive um das Verhältnis zwischen Literatur und Wissen geht (Borgards et al. 2013, 5-16), werden im Folgenden zwei als relevant betrachtet: Das Wissen in der Literatur sowie das literarische Wissen (poetische Wissensproduktion). Den dritten Ansatz, der Wissenschaft als Literatur betrachtet, um den Anteil der literarischen Mittel an der Produktion der wissenschaftlichen Erkenntnis zu untersuchen, kann man bei diesem Thema beiseitelassen.

Die politischen sowie ideengeschichtlichen Koordinaten der in der Forschung recht gut beschriebenen Absage ans geschichtsphilosophische Denken bei B. Strauß müssen hier nicht nochmal ausführlich geschildert werden, denn Strauß' allmähliche Abwendung von Adorno, Marcuse, Brecht, von links-aufklärerischer, idealistischer Prinzipialität hin zu Hamann, Heidegger, Montaigne wurde bereits überzeugend dargelegt (vgl. Windrich 2000), einschließlich der Zunahme an rechten, gegenaufklärerischen Ideologemen, die ab den 1990er Jahren registriert wurde. Um diesen Prozess nicht auf politische Begriffe reduzieren (Wende von links nach rechts), oder ihn im monokausalen Sinne auf historische Ereignisse zurückführen (etwa Absage ans linke Denken als Antwort auf den Zusammenbruch des Ostblocks) zu müssen, wird er hier erstens an einem früheren Text aus dem Jahre 1980 erfasst, und, zweitens seine Aussagen werden ins interdiskursive Spannungsfeld der naturwissenschaftlichen und politischen/historischen/literarischen Kulturen (Diskurse, Intelligenzen) eingebettet.

Hinter dem Interesse an den modernen Naturwissenschaften, das Ende der 1970er Jahre bei einigen deutschsprachigen Literaten belegt ist, dürften, egal ob es erst plötzlich aufkam, oder bereits auf eine längerfristige Beschäftigung zurückzuführen war, wie etwa bei Hans Magnus Enzensberger, der schon in den 1950er Jahren über komplexe Systeme, dissipative Strukturen, Themen der Quantenphysik, Chaostheorie, Selbstorganisation, seltsame Attraktoren, Fraktale, Bifurkationen, Isotopien, Evolution, Emergenz, Turbulenz geschrieben hatte, mindestens zwei naheliegende Gründe gestanden haben: Der dringende Wunsch kein idiot lettré zu sein - „Blamieren wird sich nur, wer hinter den kognitiven Möglichkeiten seines Jahrhunderts zurückbleibt" (Enzensberger 2004, 74), sowie das uralte und nun reaktivierte Anliegen, Kunst und Wissenschaft, Poesie und Wissen wiederum zueinander finden zu lassen. 
Die naturwissenschaftlichen Lehrjahre brachten diesem Literaten manchen ernstzunehmenden Ertrag, der vielfach auch deren politische Entwicklung beeinflusst haben mag, insofern er ihnen gerade die Untauglichkeit des geschichtsphilosophischen Paradigmas vor Augen führte (Riedel 2009, 121-132). Bei Enzensberger lautete die naturwissenschaftliche Lektion: Denke die Zukunft stets als eine womöglich offene, unabgeschlossene. Gib ihr doch - ähnlich wie der Natur - ihre Freiheit zurück, dich überraschen, ja überwältigen zu können!

\section{GEOLOGIE UM 1800 UND 1980}

Die Einsicht in die den Menschen weit überragende Komplexität der Natur, die Enzensberger eher der Beschäftigung mit moderner Physik und evolutionärer Biologie verdankte, könnte allerdings auch auf die literarische Rezeption des geologischen Wissens zurückzuführen sein. Geologie scheint in diesem Zusammenhang ein großes literarisches Potential zu besitzen, denn sie ist imstande, Raum und Zeit zu verschränken, das den Menschen unendlich überragende Alter der Natur räumlich, in Schichten, wahrnehmbar zu machen. Historisch gesehen indes, etwa in der Sattelzeit um 1800, die man für die Geburtsstunde mancher modernen Disziplinen hält, riefen die sich etablierenden geologischen Erkenntnisse.in den Menschen eher beschämende Reaktionen hervor: Geologisches Wissen soll, wissenschaftsgeschichtlich gesehen, der Menschheit eine der vier Kränkungen zugefügt haben. Neben der Kopernikanischen (Erde kein Mittelpunkt mehr), Darwin'schen (Mensch gleich Tier), Freud'schen (Mensch kein Herr im eigenen Ich-Haushalt) soll die geologische Kränkung den Menschen temporär unendlich marginalisiert haben, der auf der Erde "kurz vor zwölf“ erschienen sei, während unendliche Zeiträume der Erdgeschichte sich ohne ihn abgespielt hätten. Um den Rahmen wieder zu schließen: Zum nun offen und frei gewordenen Blick auf die nicht mehr berechenbare Zukunft, so die Lehre aus dem politisch-naturwissenschaftlichen crossing bei Enzensberger der späten 1970er Jahre, setzt die Geologie unseren (Rück)Blick dem tiefen Zeitabgrund mit anderen natürlichen (erdgeschichtlichen), das menschliche Maß überragenden Rhythmen aus, in denen der Mensch zeitlich an der Peripherie landet.

Dies dürfte auch den Einbruch der Geologie in die deutschsprachige Literatur mitbegründen, zu dem es eben um 1980 gekommen ist, allerdings ohne direkte Beteiligung von Enzensberger. 1979 sind, so Georg Braungart (2007, 23-41) gleich zwei geologisch fundierte Romane entstanden, Der Mensch erscheint im Holozän von Max Frisch, und Langsame Heimkehr von Peter Handke. Beiden Romanen ist gemeinsam, dass in ihnen die individuelle Lebensgeschichte, samt dem Tod, in die mit geologischer Begrifflichkeit erfasste Naturgeschichte eingefügt wird, deren Perspektive (sog. transhumane Perspektive) die Texte letztendlich einnehmen. In die Nähe dieser Romane wird im Folgenden auch der erste längere Prosatext von Strauß, der Roman Rumor situiert, der 1980 erschienen ist.

\section{BOTHO STRAUß: EIN UMSTRITTENER POETA DOCTUS?}

Botho Strauß gilt als poeta doctus, dessen Texten eine große Vertrautheit mit neuesten naturwissenschaftlichen Erkenntnissen anzumerken ist, deren Umsetzung al- 
lerdings in der Germanistik nicht immer einstimmig akzeptiert wurde. Strauß suchte nämlich, bereits Ende der 1970er Jahre, nach recht spekulativen Brücken zwischen Ästhetik und Naturwissenschaft, Instinkt und Vernunft. Dabei griff er auf Begriffe wie Entropie oder Emergenz zurück, um die Brücken zwischen naturwissenschaftlichen, politischen und ästhetischen Sichtweisen zu untermauern. Er agierte meist als intellektueller Synthetiker, der, so die Kritik, einzelne Begriffe aus ihren ursprünglichen Kontexten herausnehme und in neuen Konstellationen, ins metaphorische Kultur-Reservoir eingebettet, recht eigensinnig handhabe. Man hält ihm diesbezüglich vor, zwischen exakt definierten Begriffen und eher bildhafter Begrifflichkeit kaum zu unterscheiden, was ein in wissenschaftlicher Hinsicht genauso gravierender Fehlgriff sei wie etwa die dem Band Beginnlosigkeit zugrunde gelegte Hypothese, im Freiraum des literarischen Textes könnten zu Wissen auch Erkenntnisse transformiert werden, die in wissenschaftlicher Hinsicht überwunden, in den einzelnen Wissenschaftsdisziplinen als widerlegt gelten. Strauß, so der kritische Grundtenor (vgl. Hagestedt 1994), kümmere sich demnach nicht allzu sehr um den aktuellen disziplinären Wissensstand, er handhabe das disziplinäre Wissen eklektisch und dilettantisch, also recht eigensinnig und -willig.

Im Folgenden soll daher angedeutet werden, warum dieses Urteil über die vermeintliche Unwissenschaftlichkeit der Komplexität der literarischen Texte von Strauß kaum gerecht wird, die ja - wie am Beispiel von Rumor zu zeigen sein wird - die Interdiskursivität in einem modernen literarischen Sinne handhaben, um in der $\mathrm{Li}$ teratur einen wissenssubversiven Ort zu etablieren, an dem das Wissen eben gegen die Wissensspezialisierung entdifferenziert wird (vgl. Parr 2020). Gerade der Literatur, so soll gezeigt werden, möge dabei die Aufgabe zukommen, Brückenschläge über die Ränder einzelner Spezialdisziplinen hinweg vorzunehmen. Dass dabei nicht nur das wissenschaftlich up to date gebrachte Wissen literarisiert wird, ist nicht nur klar, sofern der literarische Wissensdiskurs (es kann auch nicht anders sein) von Laien, Autodidakten und Wissenssynthetikern betrieben wird, sondern - in gewissem Sinne - auch recht und gut, da nur so darin das Fachwissen frei für Umänderungen, Umakzentuierungen und Kontrafakturen gemacht werden kann, wodurch die Literatur von ästhetischer Distanz gegenüber ihrer Zeit Gebrauch macht (Riedel 2004, $350)$.

\section{INTERDISKURSIVE LEKTÜRE VON RUMOR}

Von alldem wollten allerdings die Leser des Romans Rumor lange nichts wissen. Schaut man sich dessen Rezeptionsgeschichte an, herrschten in der ersten Phase Versuche vor, diesen Roman als Geschichte eines intellektuellen Verfalls zu lesen, ein Psychogramm symptomatischer bundesrepublikanischer Intellektuellenkrise der späten 1970er Jahre. Dazu gab es gute Gründe, denn der Hauptprotagonist, Ingenieur Bekker, ein bedenklich intelligenter (an der prekären Zone zwischen Genialität und Idiotie rührender), doch immer wieder scheiternder Held, macht im Laufe des Romans in der Tat einen allmählichen Regress durch. Die ganze Zeit vor der Heimkehr ins sogenannte Institut für Nachricht stehend (abgekürzt als IfN, daher oft mit dem Frankfurter ISF assoziiert), klammert er sich, da er sich mit dem Tritt über 
die Schwelle schwertut, an seine Tochter, auch dies in einer ambivalenten Mischung aus inzestuöser Zuneigung und (Selbst)Hass eines stets versagenden Vaters. Von der Tochter wird er allerdings am Ende des Romans, am Tiefpunkt seines Verfalls, in eine separierte Wohnung abgeschoben, auf dass er sich in eine halb reale, halb mythische Gestalt zu verwandeln beginnt, die zu denken gibt. In der letzten Szene meldet sich der Vater bei seiner Tochter per Telefon, und in der „Muschel [ist] nichts anderes $\mathrm{zu}$ hören als das schwere, lüsterne, waldestiefe Atmen eines Mannes, der die Brust eines Riesen haben muß“ (Strauß [1980] 1984, 231). Als ein Riese (Titan) endet hier einer, von dessen trunksüchtigem (Silen), in seiner Vaterrolle immer wieder versagenden, nicht selten inzestuös angelegten und offensichtlich am Verhältnis zur deutschen Nation laborierenden („,waldestief“) Verhalten man sich 230 Seiten lang überzeugen kann.

In den 1990er Jahren interessierte man sich zunehmend für das zuletzt erwähnte Motiv, indem man den Spuren der nationalsozialistischen/faschistischen Nachwirkung in der Bundesrepublik nachging. In Rumor wurde nun eine prekäre Verfallsgeschichte eines Deutschland-Sohnes erblickt, Bekker galt als der bundesrepublikanische Sohn, der am nationalsozialistischen Erbe seines innerlich gebrochenen Vaters leidet, eines unehrenhaft entlassenen NS-Majors. Das Verhältnis zu seinem Vater macht Bekker zu schaffen, das Abnabeln fällt auch deshalb so schwer, da er selbst in den radikalsten Negationen zwangsläufig zur Kopie seines Vaters wird, von dem er sich absetzen will, einem „gefällten Kämpfer, rachsüchtigen und selbstherrlichen“, dessen Lebenskräfte aus „Haß, Verachtung, Vernichtungsdrang und Wille zum Tod" (19) bestehen würden, die nun der Sohn in einer vergeblichen Inversion nicht (wie der Vater) gegen Hitler, sondern gegen das System der Bundesrepublik der 1960er und 1970er Jahre richtet. In dieser Sicht wurde Rumor als Deutschlandroman, ja als Nachkriegsdeutschlandroman schlechthin gelesen. Nach und nach entdeckte man etliche intertextuelle Verweise, eine Rezeptionsphase, die bei Strauß geradezu obligatorisch ist: Namhaft gemacht wurden dabei Dante mit seiner Göttlichen Komödie, König Laer Shakespeares, Aufzeichnungen des Malte Laurids Brigge Rilkes, Theogonie Hesiods und viele andere (vgl. Kaussen 1991).

Bis heute bleibt allerdings ein wichtiger Aspekt meist ausgeklammert. All die genannten Ebenen sind in dem Roman auf eine interdiskursive Art mit dem Wissen einiger Disziplinen verflochten. Es ist in der Tat so, dass die Germanisten diesbezüglich kaum dem Vorschlag von Peter Demetz gefolgt sind, der seinerzeit auf besondere Querschnitte zwischen den Romanfiguren, der Erzählform und kosmologischen sowie erdgeschichtlichen Diskursen hingewiesen hat (Demetz 1988, 276ff); offensichtlich ein Fehler, beruht die Aussagekraft dieses Romans doch auf dem semantischen Gewebe, in dem einzelne wissenschaftliche Diskurse interagieren, um die Motive innerhalb einzelner Elemente (Erde, Feuer, Wasser, Luft) in- und übereinander wandern zu lassen, die Tore zwischen Himmel und Erde, Erdoberfläche und Erdtiefe offen haltend, mit aufschlussreichen (Erde wird anthropomorphisiert, Körpersprache wird materialisiert) literarischen Konsequenzen.

Konkret gesagt: Die erwähnten politischen und historischen Diskurse, etwa in der Form des Deutschlanddiskurses, werden in Rumor mit medizinischen, biologischen, 
psychologischen, linguistischen, soziologischen und auch geologischen Diskursen verzahnt. Realisiert wird es auf mindestens zwei Ebenen. Zunächst, vordergründiger, werden im Roman auf der Ebene der Figurenrede einige Erkenntnisse der modernen philosophisch agierenden, also populärwissenschaftlichen Biologie diskursiv referiert, meist in Paraphrasen oder Halbzitaten. So erkennt man im Disput zwischen Bekker und seinem Biologielehrer Bongie einige durch Jacques Monods Buch Le hasard et la nécessité (1970; Zufall und Notwendigkeit, 1971) populär gewordene Thesen wieder, die allerdings Bongie bereits „Mitte der fünfziger Jahre“ (Strauß [1980] 1984, 134) gekannt haben will, offenbar bevor sie dank der wissenspopularisierenden $\mathrm{Pu}$ blikation des Piper Verlags in der Bundesrepublik zur "Sachbuch-Weisheit“ (136) geworden seien: Für eine „stille Revolution des Menschenbilds“ (136) hält nun auch Bekker die Erkenntnisse der modernen Molekularbiologie, laut deren die Menschen rundum bloß mit einem „Würfelwurf hingewürfelt" (43) wurden, ein Produkt des zufälligen, durch „Tippfehler der genetischen Übertragung“ (141) entstandenen Entwicklungsprozesses darstellen - nichts für Kinder, nichts für Christen, Marxisten sowie alle anderen, die sich weigern, aus dem ideologischen Schlaf zu erwachen, so dass es Zeit ist, zu wissen, der Mensch werde, wie „Zigeuner am Rande des Universums" (141), das gleichgültig gegen seine Hoffnungen, Leiden oder Verbrechen ist, eines Tages von der Erdfläche verschwinden, zunächst von Sachen, vom Leblosen überrundet, dann zugeweht wie ein „Scheißhaufen am Strand“ (143).

Noch aufschlussreicher als die diskursiv entfalteten Szenarien einer Welt, in der das Ich zu einem Naturgeräusch geworden ist, das ,wie der Wind so durch seinen offenen Mund heult" (144), erscheint die zweite interdiskursive Ebene, auf der sich die Diskurse in ihren Aussagen quer über die Motive gegenseitig potenzieren. So wie Bekker psychisch der historische Schatten Deutschlands zusetzt, den er stets in seinem Rücken spürt, erkrankt just auch seine Tochter Grit an einer rätselhaften Krankheit, bei der sich in ihrem Rückenmark hohle Stellen bilden, auf den Röntgenbildern als dunkle Schatten sichtbar, die allerdings kaum zu heilen sind, da man, so die Ärzte, ihre Genesis nicht richtig interpretieren könne; schließlich wird ihr nahegelegt, es operativ zu probieren, um - völlig ahistorisch - in den Stamm einzugreifen. Doch auch dieser Eingriff bringt keine Besserung, so dass die Figuren letztendlich ihren diversen Schatten in historischer sowie körperlicher Form ausgeliefert bleiben, ohne Hoffnung auf jedwede bessere Zukunft, die kommen könnte, nachdem man die Schatten historisch abgearbeitet oder medizinisch auskuriert hätte. Die Parallelen und Analogien funktionieren hier nach folgendem Muster: Historische Traumata werden verinnerlicht, zu psychosomatischen Defekten transformiert, deren Spuren das individuell menschliche Maß zu übersteigen scheinen, folgt somit anderen, größeren Zeitrhythmen und sich dem menschlichen Zugriff entziehenden Logiken.

Dazu kommt noch: Soziale und emotionale Aspekte der menschlichen Verhaltens- und Kommunikationsformen werden mit Naturbegriffen und naturwissenschaftlichen Theorien assoziiert. Stets taucht man, etwa in der Arbeit, in den Äther einer stärkeren Person ein (11), Lebensenergien werden an der Wärme- und Brandskala abgemessen (glimmen, heizen, brennen für etwas oder -einander), Menschen speien vor Zorn wie qualmende Vulkane, sie stoßen einen Feuerschwall der Emoti- 
onen aus, beben, werden kalkweiß, zischen, schütten sich Aschenbrenner über ihre Haare (13). Mal geht es eher in vulkanistisch-geologische Richtung, wenn die Leute unter ihrer Brust genug Wärme vorzeigen wollen, um sich ihrer Produktivität zu vergewissern (symptomatisch auch die hohe Zahl der vom Burn-out Betroffenen), mal kreisen sie um ihre Vorgesetzten wie Planeten um die Sonne (8). Das Handeln der Protagonisten gerät mitunter via geologischer Bildhaftigkeit in längere Zeitrhythmen, mit dem Effekt, dass sie marginalisiert und ironisiert werden, oder - im Gegensatz dazu - dass es zu Zeitdilatationseffekten kommt: Die Zeit dehnt sich hier in erdgeschichtlichen Dimensionen - so etwa empfindet Bekker mit Grit „unter ihren Füßen ein berstendes Scharren, als schöben ganze Erdschilde sich übereinander" (63) - oder es kommt zu biologischen Asynchronitäten zwischen Körper und Geist: Grit kommt es beim Anblick ihres Vaters vor, als sei „ein Teil seines Wesens und auch seines Körpers in einem plötzlichen Vorsprung ins Alter vorausgestürzt" (54), später erscheint er ihr verwandelt, „wie hinübergegangen in ein anderes Lebensalter“ (84). Wiederum Grit erscheint Bekker nach ihrer ärztlichen Behandlung ,weit zurückversetzt, in ein anderes Wesen verwandelt; diesmal im Rückgang, der Klang der Stimme, die quenglige Laune sind wie die eines unartigen kleinen Mädchens" (127).

Die interdiskursiven und polyvalenten Ketten setzen bereits beim Titelwort Rumor ein: Mitgemeint dürfte das innere schmerzvoll-entbehrende Rumoren des Hauptprotagonisten-Körpers sein, seine unkontrollierten Rede- und Schimpfkanonaden, in denen sich die „Stöße des ungebändigten Lebens unter seinen Sohlen“ (50) zu Wort melden, aber auch alles, was er (individuell menschlich aber auch historisch) vermisst, was ihm Grenzen setzt, womit er nicht zu Rande kommt. Aber zugleich könnte Rumor für unbeholfene Artikulationsversuche des nationalen (nachkriegsdeutschen) Körpers stehen, genauso für die der Ordnung der Dinge zugrunde liegende Unordnung, „die immer noch unterdrückte Rede des Ganzen, ein Rumor bloß, aber überall stärker hervordringend“ (145), eine Art Zukunftsmusik der uns bevorstehenden Anarchie, in die man, nach dem Ende der Geschichte wohl hineintappen würde und deren erste Takte Bekker in seinen hellhörigsten Wahnsinnsmomenten vernimmt. Und nicht zuletzt auch, im geologischen und mythologischen Sinne, für Töne, die Bekker, ein verrückter Genius, aus dem Inneren der Erde herauszuhören vermag, wenn er vor seinen Zeitgenossen die Ohren verschließt und sich in seinen Visionen unter die Erde begibt, um im mythologischen Tartaros die mythologische Zeitlosigkeit mit den erdgeschichtlich weit zurückliegenden Zeit-Epochen und -Rhythmen kurzzuschließen.

Kurzum, der Roman bündelt unter dem Stichwort „lange Zeitrhythmen“ etliche semantische Überlappungen, wodurch er disziplinäre Grenzen durchlässig macht. An einem Beispiel demonstriert: Hinter den Rücken der Figuren wirft die Geschichte lange Schatten, in den Rückenpartien der Figuren werden die historischen Hypotheken somatisiert, in deren Rückenmark schwindet irreversibel deren lebenswichtige Substanz, hinter ihrem Rücken spielen sich unbewusste und dem Menschen unzugängliche Prozesse ab, in die man nicht hineinblicken kann, ohne es mit psychischem Rückfall zu bezahlen. Weitere Transferwörter, die hier zwischen Medizin und Geologie semantisch vermitteln, sind etwa „Rutsch“ (Rutsch der Erde, Rutsch der Kno- 
chen), folglich beide Figurennamen: Einer der Romanerzähler heißt Bruno Stöss, was semantisch zur ruckartigen Bewegung (Anprall, Stich, Schlag), zu Atem-, Wind-, oder Erddynamik (Erdstoß) sowie zu den Schichten (aufstoßen), die ja als geologischer Begriff par excellence gelten, zählt. Der Hauptprotagonist Bekker trägt in seinem Namen Spuren der künstlich (Becken als Schüssel) oder natürlich entstandenen Landschaftsform (Wasserbecken), dem die medizinischen (Becken als Körperteil, im Roman eben der zentrale Ort, wo sich das nicht Abgearbeitete ansammelt) und geologischen (Mulde der Erdoberfläche, wo Ablagerungsschichten zu sehen sind) Bedeutungen zur Seite zu stellen sind. Mit diesem interdiskursiven Transferpotenzial im Hinterkopf könnte man wohl besser als Leser oder Übersetzer mit den sonst kaum verständlichen Sätzen zurechtkommen wie: „Abwarten. Schürfen und Scharren. Der Leib ist Gestein, verschlossen der Leib, verschlossen die Sprache. Ablagerung, Verwerfung. Eine neben vielen Krusten der Erde“ (176). Hier scheint der Satz genau an der Grenze zwischen organischer und anorganischer Natur, zwischen Sprache und Körper anzusetzen, um sie durch Parallelisieren der Dimensionen der Geburt und langer Evolution sowohl der Erde als auch der einzelnen Formen der Sprache (von Menschen, Sachen, Elementen, Natur, Erde, Körper, Kunst, Mythos) verschwinden zu lassen. Die Verben „ablagern“ und „verwerfen“ liefern die entscheidenden Transfer-Kontexte mit, denn sie bezeichnen mit „ablagern“ nicht nur ein evolutionäres Abwarten (durch Lagern an Qualität gewinnen), sondern auch die geologische Sedimentierung (sich absetzen). „verwerfen“ referiert nicht nur auf die Bedeutung etwas aufgeben, etwa im medizinischen Sinne für die Fehlgeburt, sondern wiederum auch geologisch auf die gegenseitige Verschiebung von Gesteinsschichten. Abrunden lässt sich dieser interdiskursive Exkurs zu Rumor mit der letzten Romanszene, in der Grit von ihrem Vater angerufen wird, wobei die Telefonmuschel (Muschel als Medium für den fernen Transfer) ihn sehr wohl in weit zurückliegende biologische Lebensphasen mit seinen verdrängten Inzestphantasien bringt (Muschel als Vagina, oder die Ohrmuschel seiner Tochter, die er küssen will), die allerdings - via Muschel im Meer als Trilobit - nicht nur weitreichende Brücken in die geologischen, sondern auch in die mythologisch-künstlerischen Tiefen der Zeit offen halten, so etwa die Geburt der Liebesgöttin (Venus, Aphrodite) auf der Muschel aus dem Meeresschaum, wie man es aus dem bekannten Bild Botticellis kennt.

Es wundert nicht, dass dieser Roman seinen Interpreten meist rätselhaft geblieben ist. Demgegenüber konnte hier aufgezeigt werden, wie Strauß durch crossing der jeweiligen Diskurse das Genre des Deutschlandromans transformierte, indem er es in die transhumane Perspektive eingebettet hat. Dies erklärt auch die völlige Absenz jeglicher linear aufsteigenden Elemente des Bildungsromans, die selbst in den pessimistischen Varianten der Deutschlandromane in der Regel zumindest als Folie beibehalten werden. Dies hebt Rumor auch von den als Epilog nach der gescheiterten Abendlandkultur aufgefassten Bildungs- und Deutschlandromanen ab, wie sie etwa Doktor Faustus. Das Leben des deutschen Tonsetzers Adrian Leverkühn, erzählt von einem Freunde (1947) Thomas Manns repräsentiert, sofern Rumor nicht einmal den kleinen Trost bietet, der bei Mann in dem sonst recht trostlosen Schluss darin zu finden ist, dass man hier auf die qualmende Schutthalde der deutschen Unheilsge- 
schichte hochklettert, um sie von oben zu besehen, und womöglich wie Phönix aus der Asche aufzuerstehen. In Rumor gibt es keinen derartig zum neuen Anfang motivierenden Schluss: Aus den weit über den menschlichen Zugang hinaus erweiterten Prozessen gibt es weder ein Entkommen, noch kann der Mensch über sie hinauskommen, um sie rational in den Griff zu bekommen; Vorhersagen wären in diesem Roman genauso unangebracht wie Figuren, die der prägenden Last des Vergangenen entkommen würden, um neu anzufangen. Diese Subordination manifestiert sich in Rumor auch erzähltechnisch. Die langen und an sich kaum verständlichen Passagen, die Bekker erzählt, handeln nicht von irgendeinem Rumor, sie vollziehen ihn, sie sind Rumor schlechthin. Wiederum die von seinem Arbeitskollegen Stöss erzählten Passagen, dem sog. sekundären Ich-Erzähler, der in bereits klassisch gewordener Form (Stanzel 1989, 263-267) das Medium darstellt, durch das man sich dem an sich unverständlichen Rumor und dem kaum verständlichen Bekker nähern, beide doch artikulierbar machen kann, nehmen erst dann eine sinnvolle Gestalt an, sobald man sie in ihrem ineinander verzahnten und miteinander extrem synthetisch agierenden crossing der einzelnen Diskurse erkennt. Synthetisch ist dieses crossing sowohl in seiner Interdiskursivität als auch diskursintern, bringt doch Strauß, um es am geologischen Diskurs zu zeigen, sämtliche Theorien zusammen, von den neptunistischen, über die plutonistischen, vulkanistischen bis zu den plattentektonischen, um hier die individuellen Geschichten in einem übermenschlich großen Rahmen mit kaum absehbarem Anfang und offener Zukunft zu platzieren, dessen Lauf keinem Monoprinzip unterworfen ist.

\section{ERDGESCHICHTLICHE NATURMETAPHORIK IN DIE WOLFSHAUT}

Um die Eigenart von Rumor noch komplexer zu erfassen, wird ihm nun im zweiten Schritt der seinerzeit recht vergessene, erst in den 1990er Jahren von der Literaturkritik entdeckte Roman Die Wolfshaut von Hans Lebert gegenübergestellt, der im Jahre 1960 das Genre der an sich idyllischen Heimat- oder Dorfromane (Schmidt-Dengler 1990, 112) umgewandelt hat, um anhand deren Negativfolie die bis dahin kaum reflektierte Täterrolle Österreichs zum Thema zu machen. Auch hier hat man es mit einem irritierten und an seinen Heimatort recht ambivalent gebundenen Heimkehrer namens Johann Unfreund zu tun, der allerdings nicht vor den Toren des IfZ Instituts steht, sondern nach dem Krieg als Außenseiter ins Dorf namens Schweigen zurückkehrt, um aufzudecken, warum sein Vater am Kriegsende Suizid begangen hat. Auch in Die Wolfshaut findet man einen Kriegsvater-Nachkriegssohn-Fall vor, hier allerdings nicht in die dritte Enkelgeneration verlängert; Unfreund bleibt kinderlos. Und auch hier kommt das Dunkle und Ungeklärte von dem, was die Nachkriegszeit von der Kriegszeit geerbt hat; Bekkers Vater stattete seinen Sohn mit allumfassendem Hass aus, weshalb dieser in den bundesrepublikanischen Verhältnissen nie Fuß fassen konnte, Unfreunds Vater hatte sich im österreichischen Schweigen umgebracht, da er - das kommt im Laufe des Romans ans Licht - mit seiner Schuld am Massaker einiger Kriegshäftlinge nicht zu Rande kam, das er in den letzten Kriegsmonaten nahe der dörflichen Ziegelei mit einigen - bis heute lebenden - Dorfbewohnern begangen hatte. Und diese Mitverbrecher geraten zwischen November 1952 und Fe- 
bruar 1953 samt kompletter Dorfbevölkerung in eine schauderhafte Nachwirkung dieser Kriegsverbrechen, in eine genau 99 Tage lange anhaltende geradezu biblische Sintflut, die die Erde aufweicht, um sie dann mit Schnee zu bedecken. In dieser Zwischenzeit werden weitere ominöse Verbrechen verübt, deren Zweck, wie den aufmerksamen Romanlesern aber nicht den Dorfbewohnern klar wird, darin besteht, die Verbrechen der letzten Kriegstage geheim zu halten, am besten zuzudecken, wobei die vom Himmel kommenden Schneemassen mitzuspielen scheinen. Damit ist man genau an dem Punkt, wo die Menschenschicksale von den Wetterphänomenen mitbedingt werden: Menschen werden hier zum Teil einer Landschaft, welcher sich am raschesten und sichtbarsten bewegt, könnte man mit Heimito von Doderer $(1961,795)$ sagen. Es töten in diesem Roman zwar konkrete Menschen, also Täter von ehedem, doch, da sich alles im Unheimlichen und Unsichtbaren abspielt, werden als Täter vom Romanpersonal auch oder ausschließlich natürliche und übernatürliche Kräfte gemutmaßt, so etwa die entfesselten Naturkräfte wie Sturm oder Blitz, übernatürliche Instanzen wie die unter der Erde zugeschütteten und angeblich nach Rache dürstenden Opfer, oder ein bestialischer Wolf, der das Dorf eingekreist und nachts immer wieder angegriffen haben soll.

Kommt man bei Strauß dem an sich unerklärbaren und polysemantischen Rumoren erzähltechnisch mittels zwei voneinander gespaltener Personen bei, dem halb verrückten, halb genialen Bekker und seinem eher durchschnittlichen Kollegen-Medium Stöss, wird bei Lebert das unheilvolle Schweigen um die Verbrechen, das sich hier und da durch penetrante Naturzeichen (Gerüche) zu Wort meldet, auch von einem Paar-Ego gebrochen, dem als Matrose bezeichneten Unfreund und dem Fotografen Maletta. Das Dämonische braucht auch hier ein Medium, den Matrosen Unfreund, der das Böse und Dämonische (Wölfische), das im Fotografen Maletta inkarniert ist, am Romanende zur Strecke bringt, auf dass von dem eben Erschossenen - nicht ein mythologischer Riese - sondern die Wolfshaut übrigbleibt. Schlägt sich in Rumor die Belastung sogar bis in die Enkelgeneration nieder, in der sich die historischen Schatten in dunklen Flecken, tauben, verwitterten Stellen und den mit angesammeltem Restharn gefüllten Auswüchsen somatisieren, den Körper somit krank und unfruchtbar machen, nimmt in Die Wolfshaut die unverarbeitete Vergangenheit vielmehr Gestalt in einem einzigen, nämlich Malettas Körper an, worin sie sich als böses Geschwulst aufbläht, um folglich in die Natur hinein zu explodieren.

Somit ist die Natur auch hier keine Kulisse, vielmehr ein Akteur, der kaum Halt geben kann, da er in seinen Sphären (Erde, Wasser, Luft, Himmel) porös, brüchig oder doppelbödig ist. Etwa die doppelbödige Erde kann mit tatkräftiger Unterstützung des Wassers das Unterirdische (die zugeschütteten Toten, die unteren Schichten der Erde, die Eingeweiden der Erde) sozusagen an die Oberfläche hinaufspülen; oder aber ist sie zu Inversionen fähig, indem sie hinauf, in den Himmel steigt, während dieser hinabfällt, so dass es zur Durchdringung von Himmel und Erde kommt, bei der das Irdische wortwörtlich kopfstehen kann. Das Vermischen der Sphären läuft via die geologisch gefärbte Metaphorik „Schicht“ und „decken“: Die Toten werden mit der Erde zugedeckt, die Wolken bilden eine Decke, Verbrecher decken sich, Ver- 
brechen, Wahrheit wird oder wird nicht aufgedeckt u. Ä. Gerade das letzte Beispiel zeigt, wie in Leberts Roman das Gefälle - nach unten in die Erde hinein und nach oben in den Himmel hinauf - mit der zeitlichen horizontalen Linie verschränkt wird, die hier zunächst bis ins Jahr 1945 reicht. Aus der Tiefe der Erde sprechen hier die am Kriegsende brutal Ermordeten und Zugeschütteten, die Gerüche aus der Erdentiefe sind die der Verstorbenen, weshalb es auch der Erde übel wird, da sie die ungesühnten Körper der Opfer nicht mehr verdauen kann. Der Rahmen, in dem hier die Natur agiert, ist somit - anders als in Rumor - ein moralischer. Immer wenn der Matrose das Gefühl hat, das Dorf würde kopfstehen, bezeichnet es - in diesen Koordinaten - dasselbe moralische Defizit der Dorfbevölkerung, das dem Photographen Maletta signalisiert wird, der im Himmel plötzlich einen blutüberströmten Körper erkennt, in dessen Inneren die Adern platzen (Lebert 1960, 268).

Die Natur ist hier zwar weit davon entfernt, eine trostspendende dorf- oder heimatromangeschichtliche Idylle zu bieten, dennoch trägt sie, je bedrohlicher und unheilvoller sie von der Dorfbevölkerung empfunden wird, deutliche Spuren einer heilsgeschichtlichen Instanz, die nicht lockerlässt, bis die Verbrechen aufgedeckt und gesühnt werden. Dass für Signale der Natur gerade diejenigen taub und blind sind, an die diese Signale vor allem adressiert werden, also die Verbrecher von ehedem und all die, die unter die Vergangenheit einen Strich ziehen wollen, unterstreicht nur noch, dass hier die Natur als eine Weltgerichtsinstanz daherkommt, die zur Reue und Bekenntnis aufzurufen sucht, um die aus den Angeln gehobene Welt wieder geradezurücken, zumal die 1950er Jahre, in Österreich wohl noch mehr als in Deutschland, als die Jahre des kollektiven Schweigens, Verdrängens und Zudeckens bezeichnet werden.

\section{ABSCHLIESSEND ZUM KONTRAST}

Die Natur ist somit wiederum - ähnlich wie in Rumor - durch die Hauptfigur, den Unruhestifter Unfreund mit ihrer erdgeschichtlichen Vergangenheit verbunden; anders als im auf geologische Synthese angelegten Rumor überwiegen hier die neptunistischen Anklänge: Alles läuft auf die heilbringenden Wasserkräfte hinaus, aus denen sich die nun in seinen Augen unselig trocken gewordene Erde entwickelt haben soll, und deren man wiederum bedarf, um (die biblische Sintflut) die von der Erde verschlungenen und zugedeckten Verbrechen aufzudecken. Nicht die trockene Erde, sondern das zurückgekehrte Meer hält der Matrose Unfreund für seine wahre Heimat, deren Ordnung die von oben kommenden Wassermassen herbeiführen sollen. Der ungewöhnlich beständige Regen erscheint somit als Beginn einer Phase, von der aus - aus der Sicht von Unfreund - sich die Erde nicht mehr weiter entwickeln soll, es sei denn diese Entwicklung wäre eine Rückkehr des Meeres, denn nur Wasser dürfte die Erde aufweichen und die Erdbewohner womöglich zur Reue und auf den richtigen Weg bringen.

Doch diese Hoffnung bleibt im Roman unerhört. Die geologischen Ansätze werden, in Dominanz der neptunistischen Anklänge, zwar zitiert, doch da hier die Gegenwart im Zeichen der Moralabsenz als Schauplatz des Bösen daherkommt, wird der neptunistische Diskurs hier geschichtsphilosophisch zu einer Unheilsgeschichte 
umformuliert. So darf etwa die am Romanende präsente offene Muschel nicht wie in Rumor mit reicher Semantik und wertfrei präsentiert werden, sondern sie wird als „leer" bezeichnet, und ihre Leere verweist auf den leeren Himmel, in dem der in seinen Plänen gescheiterte und ins Hier und Jetzt definitiv nicht heimgekehrte Matrose nichts mehr zu sehen vermag. Das heißt: Die einzelnen diskursiven Einheiten, so etwa die geologischen Sätze, stellen in Die Wolfshaut anders als in Rumor nicht eine der vielen gleichwertigen Stimmen dar, aus deren produktiven interdiskursiven Dialog eine, linguistisch gesprochen, Satzreihe des Romans entstehen würde. Die Disziplinen stellen vielmehr untergeordnete Nebensätze dar, also subordinierende Sätze eines Satzgefüges, zu denen die große Heils- und Unheilsgeschichte den Hauptsatz und dessen großen Satzrahmen bilden. Denn die Natur ist hier in all ihren Formen, anders als in Rumor, mitnichten unberührt und dem Menschen gegenüber gleichgültig, also frei. Sie ist vielmehr ein Agent des Guten, der Böses in sich aufnimmt, um womöglich Gutes zu erzielen. Und wenn am Ende, nachdem keines der Verbrechen so richtig gesühnt wird, da der Hauptverdächtige im Nachkriegsösterreich gerade zum Landtagsabgeordneten wird, was ihm eine politische Immunität sichert, das Land nun als Ödnis, als Schlamm und Morast, ethisch sowie politisch, beschrieben wird, so hat man darin genau den Schnittpunkt, in dem hier die Naturgeschichte mit der politischen und moralischen Geschichte des Menschengeschlechts zusammenkommt. Den übergreifenden Rahmen bildet somit nicht die transhumane Perspektive der gegenüber dem menschlichen Schicksal gleichgültigen Natur, sondern die Heilsgeschichte, in der sich Mensch sowie Natur auf ein von vornherein festgelegtes geschichtsphilosophisches Ziel hinbewegen, dessen Wege ihnen beiden freilich unergründlich bleiben müssen.

\section{LITERATUR}

Borgards, Roland - Harald Neumeyer - Nicolas Pethes - Yvonne Wübben, Hrsg. 2013. Literatur und Wissen. Ein interdisziplinäres Handbuch. Stuttgart - Weimar: J. B. Metzler. DOI: https://doi. org/10.1007/978-3-476-00595-3.

Braungart, Georg. 2007. „,Katastrophen kennt allein der Mensch, sofern er sie überlebt.‘ Max Frisch, Peter Handke und die Geologie." In Figurationen der literarischen Moderne, hrsg. von Carsten Dutt Roman Luckscheiter, 23-41. Heidelberg: Universitätsverlag Winter.

Demetz, Peter. 1988. Fette Jahre, magere Jahre: deutschsprachige Literatur von 1965 bis 1985. München: Piper.

Doderer, Heimito von. 1961. „Bildnis eines Dorfes.“ Merkur 15, 162: 795-796.

Enzensberger, Hans Magnus. 1988. Mittelmaß und Wahn. Frankfurt am Main: Suhrkamp.

Enzensberger, Hans Magnus. 2004. Natürliche Gedichte. Frankfurt am Main: Insel.

Hagestedt, Lutz. 1994. „Botho Strauß: Literatur als Erkenntnis? Reflexionen aus dem beschädigten Leben der Postaufklärung." Weimarer Beiträge 40, 2: 266-281.

Kaussen, Helga. 1991. Kunst ist nicht für alle da. Zur Ästhetik der Verweigerung im Werk von Botho Strauß. Aachen: Alano.

Lebert, Hans. 1960. Die Wolfshaut. Hamburg: Claasen.

Parr, Rolf. 2020. „Interdiskurstheorie/Interdiskursanalyse.“ In Foucault-Handbuch. Leben - Werk Wirkung, 2. Auflage, hrsg. von Clemens Kammler - Rolf Parr - Ulrich Johannes Schneider, 234-237. Stuttgart: J. B. Metzler. DOI: https://doi.org/10.1007/978-3-476-05717-4_40.

Radix, Michael, eds. 1987. Strauß lesen. München - Wien: Hanser. 
Riedel, Wolfgang. 2004. „Literarische Anthropologie. Eine Unterscheidung." In Wahrnehmen und Handeln: Perspektiven einer Literaturanthropologie, hrsg. von Wolfgang Braungart - Klaus Ridder - Friedmar Apel, 337-366. Bielefeld: Aisthesis Verlag.

Riedel, Wolfgang. 2009. „Naturwissenschaft und Naturlyrik bei Hans Magnus Enzensberger.“ Zeitschrift für Germanistik 19, 1: 121-132. DOI: https://doi.org/10.3726/92124_121.

Schmidt-Dengler, Wendelin. 1990. Bruchlinien. Vorlesungen zur österreichischen Literatur 1945 bis 1990. Salzburg: Residenz.

Stanzel, Franz K. 1989. Theorie des Erzählens. Göttingen: UTB Vandenhoeck.

Strauß, Botho. [1980] 1984. Rumor. Roman. München: Deutscher Taschenbuch Verlag.

Windrich, Johannes. 2000. Das Aus für das Über. Zur Poetik von Botho Strauß Prosaband „Wohnen

Dämmern Lügen“ und dem Schauspiel „Ithaka“. Würzburg: Königshausen Neumann.

\section{Geology in the interdisciplinary "crossing" of cultures in German-language literature between 1960 and 1980: Botho Strauß and Hans Lebert}

Interdiscourse. Literature. Natural sciences. Geology. Germany. Nazism.

Taking its starting point as the fact that in modern cultures, the trend towards scientific specialization is an interdisciplinary tendency, which is being examined in the literature, the study reconstructs the scope of knowledge of Botho Strauß and Hans Lebert in the period from 1960 to 1980. Knowledge in literature and the production of knowledge in works of fiction by these authors is temporarily and thematically associated with the breakup with "Geschichtsphilosophie" at the intersection of natural sciences and cultural history. This process is located in the late 1970s, which is the time of the main interest of Strauß in natural sciences. It is followed by the interpretation of the Strauß's novel Rumor (1980), in which geology plays a major role, and Lebert's novel Die Wolfshaut (1960). The article also presents the impact that interdisciplinarity had on the genres of "Anti-Deutschlandroman" (Strauß) or Antiheimatroman (Lebert).

Doc. Mgr. Aleš Urválek, Ph.D.

Institut für Germanistik, Nordistik und Nederlandistik

Philosophische Fakultät

Masaryk Universität Brno

Arne Nováka 1

60200 Brno

Tscheschische Republik

urvalek@phil.muni.cz

ORCID: https://orcid.org/0000-0001-6307-5075 